\title{
Prediction of decannulation, oral intake recovery, overall survival and lung metastasis following oral malignant tumor resection and reconstruction
}

\author{
HIDENORI SUZUKI $^{1}$, IKUO HYODO ${ }^{2}$ and YASUHISA HASEGAWA ${ }^{1}$ \\ ${ }^{1}$ Department of Head and Neck Surgery; ${ }^{2}$ Plastic and Reconstructive Surgery, \\ Aichi Cancer Center Hospital, Nagoya, Aichi 464-8681, Japan
}

Received April 7, 2017; Accepted June 21, 2017

DOI: $10.3892 / \mathrm{ol} .2017 .7585$

\begin{abstract}
The present study investigated whether tongue base and mandibular bone defects were associated with the rate of decannulation and oral intake recovery, and survival time, including overall and lung metastasis-free survival time, in patients that underwent oral malignant tumor (OMT) resection with reconstruction. A total of 105 patients that underwent OMT resection with laryngeal preservation and reconstruction were recruited. The extent of defects was classified according to Urken's classification. The rates of decannulation and oral intake recovery were assessed with the Kaplan-Meier method. It was identified that 4-5 section segmental mandibulectomy (SM) and total glossectomy (TG) were significantly associated with a lower rate of decannulation and oral intake recovery by univariate and multivariate analysis using a Cox's proportional model. Patients in the high risk group (4-5 sections or TG) were significantly less likely to achieve decannulation and unaided oral intake. Patients in the high risk group exhibited a significantly shorter overall and lung metastasis-free survival time. Following multivariate analysis adjusted for the clinical stage (IV/I-III), past history of or postoperative radiotherapy (yes/no) and age (per year), the high risk group was associated with a significantly rate of decannulation and unaided oral intake. In conclusion, TG or wide SM is a prognostic parameter for functional and survival outcomes, including lung metastasis, in OMT.
\end{abstract}

\section{Introduction}

Oral malignant tumors (OMTs) are typically squamous cell carcinomas (SCC) or malignant melanomas; OMTs are treated

Correspondence to: Dr Hidenori Suzuki, Department of Head and Neck Surgery, Aichi Cancer Center Hospital, 1-1 Kanokoden, Chikusa, Nagoya, Aichi 464-8681, Japan

E-mail: hi.suzuki@aichi-cc.jp

Key words: oral malignant tumor, prediction, oral intake, decannulation, overall survival, lung metastasis by the surgical resection of the tumor. The reconstruction of oral cavity defects following OMT resection, including total glossectomy (TG), poses a formidable challenge for the restoration of postoperative oral function (1-12). The decannulation of a tracheostomy tube or extubation of a endotracheal tube, or restoration of oral intake function unaided by a nasogastric or gastric tube, are representative indicators of the restoration of postoperative oral function in patients that underwent OMT resection with reconstruction (1-12). Previously, we reported a significant correlation between the likelihood of restoring oral intake function and reduced extents of tongue base resection in an analysis based on 53 patients between 1993 and 2005 (1), and in 25 patients that underwent segmental mandibulectomy (SM) with reconstruction to treat mandibular bone defects between 2004 and 2011 (2). The postoperative functional outcomes for patients in conditions including TG has been well studied (3-5).

The development of lung metastasis subsequent to the treatment of OMT is associated with reduced overall survival time (13). The association between overall survival time with oral cavity defects, including TG, has also been reported (6). However, to the best of our knowledge, the association between lung metastasis and oral cavity defects has not been investigated.

The present study investigated whether oral defects following resection, including of the tongue base or mandibular bone, are associated with decannulation, oral intake recovery and survival, including lung metastasis-free survival, in patients that underwent OMT resection with reconstruction.

\section{Materials and methods}

Patients. Between January 2013 and January 2016, 109 patients, including 67 males and 33 females, with a histopathological diagnosis of primary OMT underwent tumor resection with reconstruction at the Department of Head and Neck Surgery, Aichi Cancer Center Hospital (Nagoya, Japan). Of these patients, 4 underwent tumor resection with total laryngectomy; these patients were excluded from the present study. Thus, a total of 105 patients that underwent OMT resection with laryngeal preservation and reconstruction were enrolled in the study. The study was approved by the institutional 
review board and all patients provided informed consent for the treatments and examinations.

Staging. The clinical staging of tumors was based on data from routine physical examination, nasopharyngoscopy, enhanced cervical computed tomography (CT) or magnetic resonance imaging, and 18F-2-fluorodeoxyglucose positron emission tomography with CT. The TNM classification was determined as per the International Union Against Cancer criteria (seventh edition) (14).

Treatments. All patients underwent reconstruction with or without laryngeal suspension following the en bloc resection of the primary tumor, with or without neck dissection. Neck dissection was not performed in cases of recurrent disease with a history of neck dissection. For reconstruction, a total of 96 free flaps and 11 pedicled flaps were raised. In addition, two patients had reconstructions featuring 2 flaps; the first patient underwent reconstruction with a rectus abdominus myocutaneous free flap and a pectoralis major musculocutaneous pedicled flap, and the second underwent reconstruction with a recutus abdominus myochutaneous free flap and a deltopectoral pedicled flap. Laryngeal suspension was performed in accordance with two criteria: i) The excision of the bilateral suprahyoid muscles, and ii) the presence of $\geq 50 \%$ of the tongue base, as described previously (1). With respect to airway management during surgery, 100 patients underwent tracheostomy due to postoperative bleeding, whereas 5 patients did not require tracheostomy. A nasogastric tube was inserted into all patients to allow tube feeding. Postoperative rehabilitation for the purpose of decannulation and the restoration of oral intake was assisted by a speech-language pathologist and nurses, as previously described (1). A total of 26 patients underwent postoperative radiotherapy, with or without platinum-based chemotherapy, due to the presence of a positive surgical margin, multiple lymph node metastases or extranodal tumor spread. The remaining methods for postoperative treatment and follow-up protocols have been previously described (14).

Defects due to tumor resection. The extent of three anatomical defects, including the tongue base, mobile tongue and mandible bone, due to tumor resection was classified with Urken's classification, as previously described (2,7). Bone defects resulting from SM were described with combinations of the letter $\mathrm{C}$ (condyle), R (ramus), B (bony) and S (symphysis) (2,7), and the number of defects was numbered 0-5 based on the number of sections removed.

Clinical parameters. The following clinical parameters were extracted from the medical records of the patients: Age, sex, clinical $\mathrm{T}$ and $\mathrm{N}$ classification, clinical stage, tumor site (tongue or other), pathological diagnosis (SCC or other), recurrence status, history of radiotherapy and surgery, induction chemotherapy, extent of mobile tongue resection, extent of tongue base resection, section of SM (section 0/1-5), skin resection, lateral pharynx resection and laryngeal suspension status, type of neck dissection (unilateral or bilateral), reconstruction flap (free or pedicle), tracheostomy indication, smoking and alcohol consumption status, Charlson comorbidity index (CCI; a weighted index based on 19 comorbid conditions) (15), body mass index, American Society of Anesthesiologists (ASA) score (16), \% vital capacity, forced expiratory rate per $1 \mathrm{sec}$ (FEV1\%), forced expiratory volume per $1 \mathrm{sec}$ (FEV1.0) and postoperative radiotherapy status.

Statistical analysis. Statistical analysis was performed using the JMP software package (version 9; SAS Institute, Cary, $\mathrm{NC}$, USA). The associations between tracheostomy indication (presence/absence) and clinical parameters were assessed using a Mann-Whiney $U$ or $\chi^{2}$ test. Applying the method described previously (1), the proportion of patients that achieved decannulation and the restoration of unaided oral intake following surgery was calculated by the Kaplan Meier method; the duration was defined as the period from surgery to the target event, or until the date of last contact. The target events were: Free of tracheostomy tube, or extubation of endotracheal tube, for decannulation; free of tube feeding, including nasogastric or gastric tube, for oral intake recovery. Oral intake was defined as the ability to intake a limited diet, e.g., soft diet, without tube feeding. Applying a modified version of a previous method $(14,15)$, various cutoff values for decannulation following resection by SM and tongue base resection were tested by univariate survival analysis, performed using Cox's proportional model. A multivariate analysis was performed to assess the clinical parameters associated with decannulation and oral intake recovery. The associations of the high (SM of 4-5 sections and/or TG) and low risk (SM of 0-3 sections and no TG) groups with clinical parameters was assessed using a Mann Whitney $U$ or $\chi^{2}$ test. A multivariate analysis adjusted for clinical stage (IV/I-III), past history of or postoperative radiotherapy (yes/no) and age (per 1 year) was performed to investigate the factors associated with both decannulation and oral intake recovery. Differences between groups in overall, local recurrence-free, regional recurrence-free, distant metastasis-free, and lung metastasis-free survival time were assessed by a Wilcoxon test (14). The associations between decannulation, oral intake recovery and lung metastasis were assessed using a $\chi^{2}$ test. $\mathrm{P}<0.05$ was considered to indicate a statistically significant difference.

\section{Results}

Patient characteristics. The clinical characteristics of the patients are listed in Table I.

Sites of the primary tumor were as follows: Tongue, 48; lower gum, 35; cheek mucosa, 9; floor of mouth, 7; upper gum, 3; mandible bone, 2; lip, 1. Histological classifications of the primary tumors were as follows: SCC, 95; malignant melanoma, 3; osteosarcoma, 2; undifferentiated sarcoma, 2; adenoid cystic carcinoma, 1; adenocarcinoma (not otherwise specified), 1; rhabdomyosarcoma, 1 .

Tongue base defects were as follows: None, 66; one-quarter of tongue base, 11; one-half of tongue base, 12; three-quarters of tongue base, 10; TG, 6. Mobile tongue defects were as follows: None, 43; one-quarter of mobile tongue, 8 ; one-half of tongue base, 15; three-quarters of mobile tongue, 17; total mobile tongue resection, 4 .

A total of 42 patients had bone defects from SM, as follows: B, 2; RB, 18; BS, 1; CRB, 2; RBS, 9; RBSB, 6; RBSBR, 4. Patients were grouped by the extent of the bone defect as 
Table I. Clinical parameters.

A, Associations between patient characteristics with tracheostomy indications and resection risk, as determined with the Mann-Whitney U test

\begin{tabular}{|c|c|c|c|c|c|c|c|}
\hline \multirow[b]{2}{*}{ Parameter } & \multirow[b]{2}{*}{ Total, n } & \multicolumn{2}{|c|}{$\begin{array}{l}\text { Tracheostomy } \\
\text { indication }\end{array}$} & \multirow[b]{2}{*}{ P-value } & \multicolumn{2}{|c|}{ Resection group } & \multirow[b]{2}{*}{ P-value } \\
\hline & & Presence & Absence & & High risk & Low risk & \\
\hline Sex & & & & 0.34 & & & 0.77 \\
\hline Male & 69 & 67 & 2 & & 10 & 59 & \\
\hline Female & 36 & 33 & 3 & & 6 & 30 & \\
\hline T stage & & & & 0.18 & & & 0.03 \\
\hline $\mathrm{T} 1$ & 2 & 2 & 0 & & 2 & 36 & \\
\hline $\mathrm{T} 2$ & 27 & 24 & 3 & & & & \\
\hline $\mathrm{T} 3$ & 9 & 8 & 1 & & & & \\
\hline $\mathrm{T} 4$ & 67 & 66 & 1 & & 14 & 53 & \\
\hline $\mathrm{N}$ stage & & & & 0.64 & & & 0.34 \\
\hline No & 51 & 47 & 4 & & 6 & 45 & \\
\hline N1 & 12 & 12 & 0 & & 10 & 44 & \\
\hline $\mathrm{N} 2 \mathrm{a} / \mathrm{b}$ & 25 & 24 & 1 & & & & \\
\hline $\mathrm{N} 2 \mathrm{c}$ & 16 & 16 & 0 & & & & \\
\hline N3 & 1 & 1 & 0 & & & & \\
\hline Clinical stage & & & & 0.044 & & & 0.10 \\
\hline I & 1 & 1 & 0 & & 2 & 26 & \\
\hline II & 20 & 17 & 3 & & & & \\
\hline III & 7 & 6 & 1 & & & & \\
\hline IV & 77 & 76 & 1 & & 14 & 63 & \\
\hline Tumor site & & & & 1.00 & & & 0.86 \\
\hline Tongue & 48 & 46 & 2 & & 7 & 41 & \\
\hline Other & 57 & 54 & 3 & & 9 & 48 & \\
\hline Pathological diagnosis & & & & 1.00 & & & 1.00 \\
\hline Squamous cell carcinoma & 95 & 90 & 5 & & 15 & 80 & \\
\hline Other & 10 & 10 & 0 & & 1 & 9 & \\
\hline Recurrence status & & & & 0.18 & & & 0.77 \\
\hline New & 72 & 70 & 2 & & 12 & 29 & \\
\hline Recurrence & 33 & 30 & 3 & & 4 & 60 & \\
\hline Radiotherapy & & & & 0.28 & & & 0.81 \\
\hline Yes & 22 & 20 & 2 & & 3 & 19 & \\
\hline No & 83 & 80 & 3 & & 13 & 70 & \\
\hline Surgery & & & & 0.12 & & & 0.55 \\
\hline Yes & 28 & 25 & 3 & & 3 & 25 & \\
\hline No & 77 & 75 & 2 & & 13 & 64 & \\
\hline Induction chemotherapy & & & & 0.16 & & & 0.06 \\
\hline Yes & 37 & 37 & 0 & & 9 & 28 & \\
\hline No & 68 & 63 & 5 & & 7 & 61 & \\
\hline Mobile tongue resection & & & & 0.22 & & & $<0.01$ \\
\hline None & 43 & 39 & 4 & & 1 & 42 & \\
\hline $1 / 4$ & 8 & 7 & 1 & & 15 & 47 & \\
\hline $1 / 2$ & 15 & 15 & 0 & & & & \\
\hline $3 / 4$ & 17 & 17 & 0 & & & & \\
\hline
\end{tabular}


Table I. Continued.

A, Associations between patient characteristics with tracheostomy indications and resection risk, as determined with the Mann-Whitney U test

\begin{tabular}{|c|c|c|c|c|c|c|c|}
\hline \multirow[b]{2}{*}{ Parameter } & \multirow[b]{2}{*}{ Total, $\mathrm{n}$} & \multicolumn{2}{|c|}{$\begin{array}{l}\text { Tracheostomy } \\
\text { indication }\end{array}$} & \multirow[b]{2}{*}{ P-value } & \multicolumn{2}{|c|}{ Resection group } & \multirow[b]{2}{*}{ P-value } \\
\hline & & Presence & Absence & & High risk & Low risk & \\
\hline Total & 22 & 22 & 0 & & & & \\
\hline Tongue base resection & & & & 0.54 & & N/A & \\
\hline None & 66 & 61 & 5 & & & & \\
\hline $1 / 4$ & 11 & 11 & 0 & & & & \\
\hline $1 / 2$ & 12 & 12 & 0 & & & & \\
\hline $3 / 4$ & 10 & 10 & 0 & & & & \\
\hline Total glossectomy & 6 & 6 & 0 & & & & \\
\hline Sections of segmental mandibulectomy & & & & 0.05 & & N/A & \\
\hline 0 & 63 & 61 & 3 & & & & \\
\hline 1 & 2 & 1 & 1 & & & & \\
\hline 2 & 19 & 19 & 0 & & & & \\
\hline 3 & 11 & 10 & 1 & & & & \\
\hline 4 & 6 & 6 & 0 & & & & \\
\hline 5 & 4 & 4 & 0 & & & & \\
\hline Skin resection & & & & 1.00 & & & $<0.01$ \\
\hline Yes & 19 & 18 & 1 & & 7 & 12 & \\
\hline No & 86 & 82 & 4 & & 9 & 77 & \\
\hline Lateral pharynx resection & & & & 0.32 & & & 0.77 \\
\hline Yes & 32 & 32 & 0 & & 4 & 28 & \\
\hline No & 73 & 68 & 5 & & 12 & 61 & \\
\hline Laryngeal suspension & & & & 0.08 & & & $<0.01$ \\
\hline Yes & 43 & 43 & 0 & & 16 & 17 & \\
\hline No & 62 & 57 & 5 & & 0 & 62 & \\
\hline Type of neck dissection & & & & 0.19 & & & 0.14 \\
\hline None & 11 & 10 & 1 & & 0 & 11 & \\
\hline Unilateral & 54 & 50 & 4 & & & & \\
\hline Bilateral & 40 & 40 & 0 & & 16 & 78 & \\
\hline Reconstruction flap & & & & $<0.01$ & & & 0.60 \\
\hline Free & 97 & 95 & 2 & & 16 & 81 & \\
\hline Pedicle & 8 & 5 & 3 & & 0 & 8 & \\
\hline Tracheostomy indication & & N/A & & & & & 1.00 \\
\hline Yes & 100 & & & & 16 & 84 & \\
\hline No & 5 & & & & 0 & 5 & \\
\hline Smoking & & & & 0.07 & & & 0.28 \\
\hline Smoker & 65 & 64 & 1 & & 12 & 53 & \\
\hline Non-smoker & 40 & 36 & 4 & & 4 & 36 & \\
\hline Alcohol & & & & 0.17 & & & 0.24 \\
\hline Drinker & 58 & 57 & 1 & & 11 & 47 & \\
\hline Non-drinker & 47 & 43 & 4 & & 5 & 42 & \\
\hline History of or postoperative radiotherapy & & & & 1.00 & & & 0.10 \\
\hline Yes & 46 & 44 & 2 & & 10 & 36 & \\
\hline No & 59 & 56 & 3 & & 6 & 53 & \\
\hline
\end{tabular}


Table I. Continued.

$\mathrm{B}$, Associations between patient characteristics (as mean \pm standard deviation) with tracheostomy indications and resection risk, as determined with the $\chi^{2}$ test

\begin{tabular}{|c|c|c|c|c|c|c|c|}
\hline \multirow[b]{2}{*}{ Parameter } & \multirow[b]{2}{*}{ All patients } & \multicolumn{2}{|c|}{ Tracheostomy indication } & \multirow[b]{2}{*}{ P-value } & \multicolumn{2}{|c|}{ Resection group } & \multirow[b]{2}{*}{ P-value } \\
\hline & & Presence & Absence & & High risk & Low risk & \\
\hline Age & $57.3 \pm 16.6$ & $57.2 \pm 16.8$ & $59.0 \pm 12.4$ & 0.98 & $57.1 \pm 21.4$ & $57.3 \pm 15.7$ & 0.57 \\
\hline Charlson comorbidity index & $1.37 \pm 1.61$ & $1.38 \pm 1.64$ & $1.20 \pm 0.84$ & 0.80 & $1.38 \pm 1.36$ & $1.37 \pm 1.65$ & 0.78 \\
\hline Body mass index $\left(\mathrm{kg} / \mathrm{m}^{2}\right)$ & $20.8 \pm 3.36$ & $20.9 \pm 3.31$ & $18.3 \pm 3.42$ & 0.12 & $19.8 \pm 3.30$ & $21.0 \pm 3.35$ & 0.10 \\
\hline ASA-PS & $1.63 \pm 0.52$ & $1.64 \pm 0.52$ & $1.40 \pm 0.55$ & 0.32 & $1.88 \pm 0.34$ & $1.58 \pm 0.54$ & 0.03 \\
\hline Vital capacity (\%) & $102.9 \pm 80.3$ & $103.2 \pm 82.2$ & $97.6 \pm 18.3$ & 0.79 & $95.3 \pm 11.9$ & $104.2 \pm 86.6$ & 0.82 \\
\hline FEV1\% (\%) & $81.7 \pm 8.69$ & $81.1 \pm 8.84$ & $79.2 \pm 4.67$ & 0.32 & $79.6 \pm 9.39$ & $82.0 \pm 8.57$ & 0.26 \\
\hline FEV1.0 (l) & $2.61 \pm 0.82$ & $2.63 \pm 0.82$ & $2.30 \pm 0.81$ & 0.39 & $2.50 \pm 0.78$ & $2.63 \pm 0.83$ & 0.60 \\
\hline
\end{tabular}

ASA-PS, American Society of Anesthesiologists Physical Status; FEV1\%, forced expiratory rate in 1 sec; FEV1.0, forced expiratory volume in $1 \mathrm{sec}$.

follows: 0 sections (i.e., no $\mathrm{SM}, \mathrm{n}=63), 1$ section $(\mathrm{n}=2 ; \mathrm{B}, 2), 2$ sections ( $\mathrm{n}=19$; RB, 18; BS, 1$), 3$ sections ( $\mathrm{n}=11$; CRB, 2; RBS, 9), 4 sections ( $n=6$; RBSB, 6), 5 sections ( $n=4$; RBSBR, 4).

Tracheostomy and follow-up. Indications for tracheostomy were present in $95.2 \%(100 / 105)$ of the study population. The association between clinical parameters and tracheostomy indication is described in Table I. Positive indication for tracheostomy was significantly associated with a higher clinical stage $(\mathrm{P}<0.05)$ and free flap reconstruction $(\mathrm{P}<0.01)$. In the entire study population, $93(88.6 \%)$ and $84(80 \%)$ were able to achieve decannulation and oral intake, respectively. The mean \pm standard deviation (SD) durations of follow-up for the entire study population, for the $78(74.3 \%)$ surviving patients and for the $27(25.7 \%)$ deceased patients were $486 \pm 300$, $526 \pm 313$ and $369 \pm 225$ days, respectively.

Decannulation and oral intake recovery by Cox's proportional hazards model. P-value and various cutoff values of decannulation for the extent of SM and tongue base resection, tested in a univariate analysis using Cox's proportional hazards model, are included in Fig. 1. Based on a univariate analysis, 4-5 section SM $(n=10)$ was significantly associated with a lower rate of decannulation $(\mathrm{P}<0.01)$ and the recovery of oral intake $(\mathrm{P}<0.01)$. Furthermore TG $(\mathrm{n}=6)$ was significantly associated with a lower rate of decannulation $(\mathrm{P}<0.001)$ and oral intake recovery $(\mathrm{P}<0.05$; Fig. 2$)$. Of the 6 patients that underwent $\mathrm{TG}$, $1(16.7 \%)$ and $4(66.7 \%)$ patients were able to achieve decannulation and oral intake, respectively. Of the 10 patients that underwent SM (4-5 sections), 4 (40.0\%) and 6 (60.0\%) patients were able to achieve decannulation and oral intake, respectively. The results of multivariate analysis, subsequent to adjusting for the extent of SM (0-3 vs. 4-5 sections of SM) and tongue base resection (non-TG vs. TG), are included in Table II. The SM of 4-5 sections was significantly associated with a lower rate of decannulation $(\mathrm{P}<0.02)$ and oral intake recovery $(\mathrm{P}<0.01)$; TG was significantly associated with a lower rate of decannulation $(\mathrm{P}<0.01)$ and oral intake recovery $(\mathrm{P}<0.01)$.
High and low risk groups. The patients were divided into high (4-5 sections $\mathrm{SM}$ and/or TG, $\mathrm{n}=16$ ) and low risk (0-3 sections $\mathrm{SM}$ and no TG, $\mathrm{n}=89$ ) groups. From the high risk group, 5 $(31.3 \%)$ and $10(62.5 \%)$ patients were able to achieve decannulation and unaided oral intake, respectively. In the low risk group, 79 (88.8\%) and $83(93.3 \%)$ achieved decannulation and oral intake, respectively. For the 5 patients in the high risk group that achieved decannulation, the mean \pm SD period from the initial surgery to decannulation was $87.2 \pm 84.71$ days. For the 79 patients in the low risk group that achieved decannulation, the mean \pm SD period from the initial operation to decannulation was $43.73 \pm 86.51$ days. Patients in the high risk group had a significantly lower rate of decannulation $(\mathrm{P}<0.0001)$ and oral intake recovery $(\mathrm{P}<0.0002)$ in the univariate analysis (Fig. 3). The association between the clinical parameters with the two groups (high and low risk) are included in Table I. Patients in the low risk group were less likely to have undergone mobile tongue resection $(\mathrm{P}<0.01)$, skin resection $(\mathrm{P}<0.01)$ or laryngeal suspension $(\mathrm{P}<0.01)$ than those in the high risk group.

Multivariate analysis for decannulation and oral intake recovery. The results of the multivariate analysis for decannulation and oral intake recovery, performed subsequent to adjusting for clinical stage (IV/I-III), past history of or postoperative radiotherapy (yes/no) and age (per 1 year), is included in Table III. The high risk group exhibited a significantly lower rate of decannulation $(\mathrm{P}<0.01)$ and oral intake recovery $(\mathrm{P}<0.01)$. Patients with a history of radiotherapy or postoperative radiotherapy were less likely to recover oral intake $(\mathrm{P}<0.03)$.

Survival. A total of 10 patients were diagnosed with lung metastasis by imaging, and 5 by pathological analysis. Patients in the high risk groups exhibited significantly shorter overall $(\mathrm{P}<0.05)$ and lung metastasis-free $(\mathrm{P}<0.02)$ survival time than the low risk groups. However, patients in the high risk group did not exhibit a significant difference in local recurrence free $(P=0.78)$, regional recurrence free $(P=0.94)$ or distant metastasis free $(\mathrm{P}=0.0515)$ survival time. The Kaplan Meier 

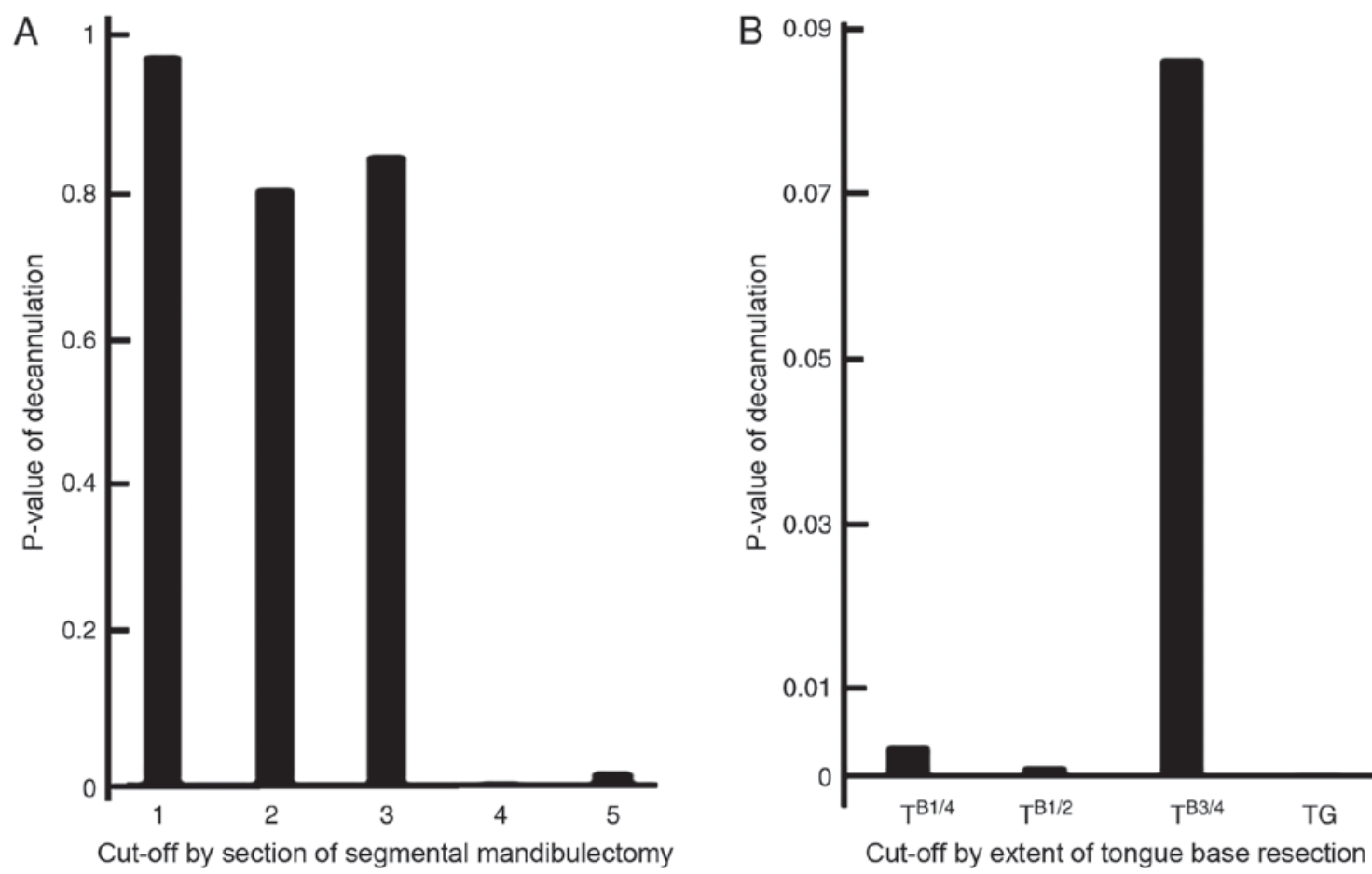

Figure 1. P-values from Cox's proportional hazard model for the rate of decannulation in 105 patients with oral malignant tumors. (A) Likelihood of decannulation subsequent to segmental mandibulectomy by the extent of resection. (B) Likelihood of decannulation subsequent to tongue base resection by the extent of resection. $\mathrm{T}^{\mathrm{B}}{ }_{1 / 4}$, one-quarter tongue base resection; $\mathrm{T}_{1 / 2}^{\mathrm{B}}$, one-half tongue base resection; $\mathrm{T}_{3 / 4}^{\mathrm{B}}$, three-quarters tongue base resection; $\mathrm{TG}$, total glossectomy.

A
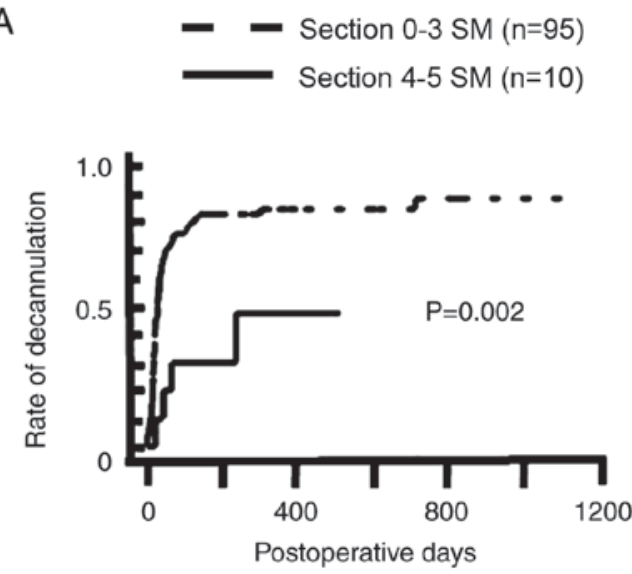

C
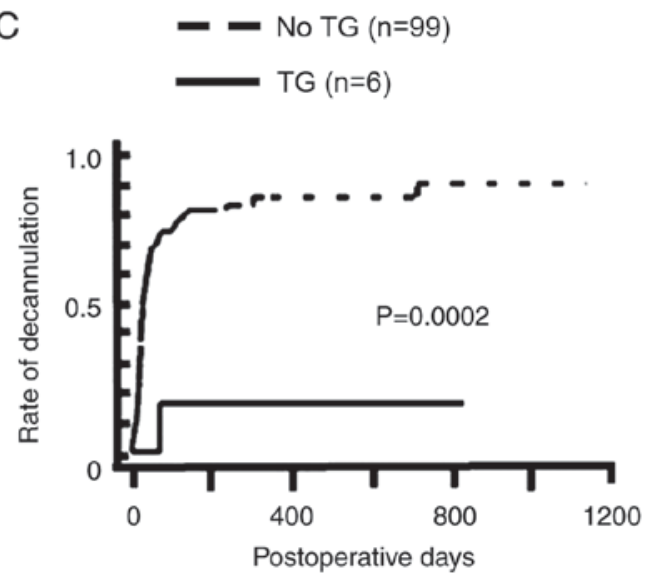

B


D

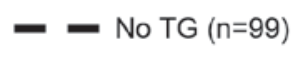

TG $(n=6)$

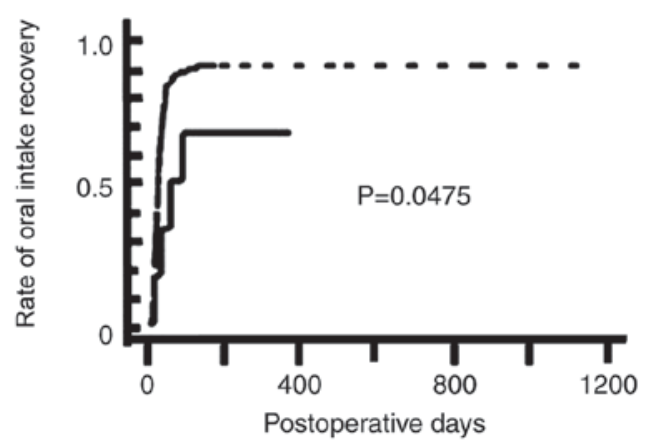

Figure 2. Kaplan Meier analysis of the cumulative probability of decannulation and oral intake recovery. Patients that underwent 4-5 section segmental mandibulectomy exhibited a significantly lower rate of (A) decannulation and (B) oral intake recovery compared with patients that underwent $0-3$ section segmental mandibulectomy. Patients with TG exhibited lower rates of (C) decannulation and (D) oral intake recovery compared with patients with no TG. Cox's proportional hazard model was used for statistical analysis. TG, total glossectomy. 
Table II. Multivariate analysis adjusted for tongue base resection and segmental mandibulectomy.

\begin{tabular}{|c|c|c|c|c|c|c|}
\hline \multirow[b]{2}{*}{ Parameter } & \multicolumn{3}{|c|}{ Oral intake recovery } & \multicolumn{3}{|c|}{ Decannulation } \\
\hline & HR & $95 \% \mathrm{CI}$ & P-value & HR & $95 \% \mathrm{CI}$ & P-value \\
\hline Tongue base resection, TG vs. non-TG & 0.35 & $0.11-0.85$ & 0.018 & 0.08 & $0.004-0.34$ & $<0.01$ \\
\hline Sections of segmental mandibulectomy, 4-5 vs. 0-3 & 0.32 & $0.12-0.67$ & $<0.01$ & 0.23 & $0.07-0.56$ & $<0.01$ \\
\hline
\end{tabular}

HR, hazard ratio; CI, confidence interval; TG, total glossectomy.

Table III. Multivariate analysis adjusted for resection group, clinical stage, radiotherapy status and age.

\begin{tabular}{|c|c|c|c|c|c|c|}
\hline \multirow[b]{2}{*}{ Parameter } & \multicolumn{3}{|c|}{ Decannulation } & \multicolumn{3}{|c|}{ Oral intake recovery } \\
\hline & HR & $95 \% \mathrm{CI}$ & P-value & HR & $95 \% \mathrm{CI}$ & P-value \\
\hline Resection group, high vs. low risk & 0.17 & $0.06-0.38$ & $<0.01$ & 0.37 & $0.18-0.68$ & $<0.01$ \\
\hline Clinical stage, IV vs. I-III & 0.61 & $0.38-1.01$ & 0.05 & 0.81 & $0.52-1.33$ & 0.40 \\
\hline Past history or postoperative radiotherapy, yes/no & 0.67 & $0.42-1.05$ & 0.08 & 0.60 & $0.39-0.93$ & 0.022 \\
\hline Age per 1 year & 0.99 & $0.98-1.00$ & 0.14 & 0.99 & $0.98-1.00$ & 0.05 \\
\hline
\end{tabular}


Figure 3. Kaplan Meier analysis of the cumulative probability of decannulation and oral intake recovery. Patients in the high risk group exhibited significantly lower rates of (A) decannulation and (B) oral intake recovery compared with patients in the low risk group. Cox's proportional hazard model was used for statistical analysis.


Figure 4. Kaplan Meier analysis of the cumulative probability of overall and lung metastasis-free survival. Patients in the high risk group (n=16) exhibited a significantly reduced rate of (A) overall and (B) lung metastasis-free survival compared with patients in the low risk group ( $\mathrm{n}=89$ ), as determined by a Wilcoxon test. 
curves for the overall and lung-metastasis free survival times are included in Fig. 4. A significant association was observed between decannulation and oral intake recovery $(\mathrm{P}<0.01)$; however, lung metastasis was not significantly associated with decannulation $(\mathrm{P}=0.17)$ or oral intake recovery $(\mathrm{P}=0.37)$.

\section{Discussion}

In the present study, it was demonstrated for the first time that patients that underwent OMT resection (SM of 4-5 sections or TG) had a significantly lower rate of decannulation $(\mathrm{P}<0.0001)$ and oral intake recovery $(\mathrm{P}<0.0002)$, as well as shorter overall $(\mathrm{P}<0.05)$ and lung metastasis-free $(\mathrm{P}<0.02)$ survival time.

Results of a national survey in the United Kingdom revealed that $69 \%$ of clinical units performed tracheostomy 'usually' or 'almost always' following free flap surgery (8). Lee et al (9) reported in 2015 that tracheostomy is commonly performed to secure the airway after oral cancer resection with reconstruction. The data from the present study, that $95.2 \%$ of the patients had an indication for tracheostomy, is consistent with those reports $(8,9)$.

Additionally, consistent with the previous reports of associations between functional outcomes and TG $(3,4)$, TG was significantly associated with a lower rate of decannulation in the 105 patients that underwent head and neck cancer resection with larynx preservation and reconstruction (3), and the functional outcomes, including oral intake recovery following subtotal glossectomy, were superior compared with TG in a review of TG without laryngectomy (4). All these findings indicate that there is an association of TG with a lower rate of decannulation and oral intake recovery $(3,4)$.

A total of 6 of the 7 patients that underwent reconstruction following large composite resection with hemimandibulectomy remained dependent on tube-feeding, which is also consistent with a previous report of functional outcomes subsequent to SM (10). Additionally, the observation from the present study of a significant association between the SM of 4-5 sections and a lower rate of oral intake recovery is consistent with the previous study (10).

The lack of uniformity in measurements of oral function subsequent to head and neck surgery with reconstruction has been discussed in several reviews $(11,12)$. Therefore, the present study functionally measured oral intake and decannulation using the Kaplan-Meier method, and assessed the association using Cox's proportional hazards model, in subjects that underwent OMT resection, as described in a previous study in which oral intake was functionally measured in subjects that underwent tongue base resection (1).

Patients that undergo TG or the SM of 4-5 sections exhibit poor overall survival time $(6,10)$. The present study, in addition to previous studies, reports a direct association between lung metastasis and shorter overall survival time in patients with OMT $(13,14)$. We hypothesized that the association of TG or 4-5 sections of SM with reduced survival time may be associated with lung metastases, as the lung is the most common site for distant metastasis in OMT (14). Accordingly, in the present study, patients with TG or 4-5 sections of SM exhibited a significantly shorter overall and lung metastasis free survival time. Thus, TG or 4-5 sections of SM may be a novel prognostic marker for lung metastasis in OMT. In addition, the results of the present study suggested that offering OMT resection with laryngectomy to patients undergoing 4-5 section SM or TG with reconstruction may be advisable; patients from the high risk group exhibited a significantly decreased rate of decannulation and oral intake recovery compared with that exhibited by those from the low risk group (0-3 sections SM and no TG).

The small number of subjects and the retrospective design are key limitations of the present study. Future studies with increased sample sizes may provide more robust results.

In conclusion, the present study demonstrated for the first time that high risk patients (SM of 4-5 sections or TG) undergoing OMT resection with reconstruction exhibit a significantly lower rate of decannulation $(\mathrm{P}<0.0001)$ and oral intake recovery $(\mathrm{P}<0.0002)$, in addition to shorter reduced overall $(\mathrm{P}<0.05)$ and lung metastasis-free $(\mathrm{P}<0.02)$ survival time, when compared with low risk patients (SM of 0-3 sections and no TG). These results suggest that TG or 4-5 section SM are prognostic parameters for both functional and survival outcomes, including lung metastasis, for individuals with OMT.

\section{Acknowledgements}

The present study was supported by JSPS KAKENHI (grant no. 16K11253).

\section{References}

1. Fujimoto Y, Hasegawa Y, Yamana H, Ando A and Nakashima T: Swallowing function following extensive resection of oral or oropharyngeal cancer with laryngeal suspension and cricopharyngeal myotomy. Laryngoscope 117: 1343-1348, 2007.

2. Mizukami T,Hyodo I, Fukamizu H and Mineta H: Reconstruction of lateral mandibular defect: A comparison of functional and aesthetic outcomes of bony reconstruction vs soft tissue reconstruction long-term follow-up. Acta Otolaryngol 133: 1304-1310, 2013.

3. Isaac A, Zhang H, Varshney S, Hamilton S, Harris JR, O'Connell DA, Biron VL and Sekaly H: Predictors of Failed and Delayed Decannulation after Head and Neck Surgery. Otolaryngol Head Neck Surg 155: 437-442, 2016.

4. Rigby MH and Hayden RE: Total glossectomy without laryngectomy-a review of functional outcomes and reconstructive principles. Curr Opin Otolaryngol Head Neck Surg 22: 414-448, 2014.

5. Dziegielewski PT, Ho ML, Rieger J, Singh P, Landgille M, Harris JR and Seikaly H: Total glossectomy with laryngeal preservation and free flap reconstruction: Objective functional outcomes and systemic review of the literature. Laryngoscope 123: 140-145, 2013.

6. Chang EI, Yu P, Skoracki RJ, Liu J and Hanasono MM. Comprehensive analysis of functional outcomes and survival after microvascular reconstruction of glossectomy defects. Ann Surg Oncol 22: 3061-3069, 2015.

7. Urken ML, Weinberg H, Vickery C, Bunchbinder D, Lawson W and Biller HF: Oromandibular reconstruction using microvascular composite free flaps. Report of 71 cases and a new classification scheme for bony, soft-tissue, and neurologic defects. Arch Otolaryngol Head Neck Surg 117: 733-744, 1991.

8. Marsh M, Elliott S, Anand R and Brennan PA: Early postoperative care for free flap head \& neck reconstructive surgery a national survey of practice. Br J Oral maxillofac Surg 47: 182-185, 2009.

9. Lee HJ, Kim JW, Choi SY, Kim CS, Kwon TG and Paeng JY: The evaluation of a scoring system in airway management after oral cancer surgery. Maxillofac Plast Reconstr Surg 37: 19, 2015.

10. Butler CE and Lewin JS: Reconstruction of large composite oromandibulomaxillary defects with free vertical recuts abdominis myocutaneous flaps. Plast Reconstr Surg 113: 499-507, 2004. 
11. Kreeft AM, van der Molen L, Hilgers FJ and Baim AJ: Speech and swallowing after surgical treatment of advanced oral and oropharyngeal carcinoma: A systematic review of the literature. Eur Arch Otorhinolaryngol 266: 1687-1698, 2009.

12. Rathod S, Livergant J, Klein J, Witterick I and Ringash J: A systemic review of quality life in head neck cancer treated with surgery with without adjuvant treatement. Oral Oncol 51: 888-900, 2015

13. Shiiba M, Unozawa M, Higo M, Kouzu Y, Kasamatsu A, Sakamoto Y, Ogawara K, Uzawa K, Takiguchi Y and Tanzawa H: Controlling distant metastasis and surgical treatment are crucial for improving clinical outcome in uncommon head and neck malignancies, such as non-squamous carcinoma. Mol Clin Oncol 2: 609-617, 2014
14. Suzuki H, Beppu S, Hanai N, Hirakawa H and Hasegawa Y: Lymph node density predicts lung metastasis in oral squamous cell carcinoma. Br J Oral Maxillofac Surg 54: 213-218, 2016.

15. Suzuki H, Hanai N, Nishikawa D, Fukuda Y, Koide Y, Kodaira T, Tachibana H, Tomita N, Makita C and Hasegawa Y: The Charlson comorbidity index is a prognostic factor in sinonasal tract squamous cell carcinoma. Jpn J Clin Oncol 46: 646-651, 2016.

16. Coskunfirat OK, Chen HC, Spanio S and Tang YB: The safety of microvascular free tissue transfer in the elderly population. Plast Reconstr Surg 115: 771-775, 2005. 